\section{INFECTIOUS DISEASE}

\section{Towards poliovirus eradication}

N. Engl. J. Med. 379, 834-845 (2018).

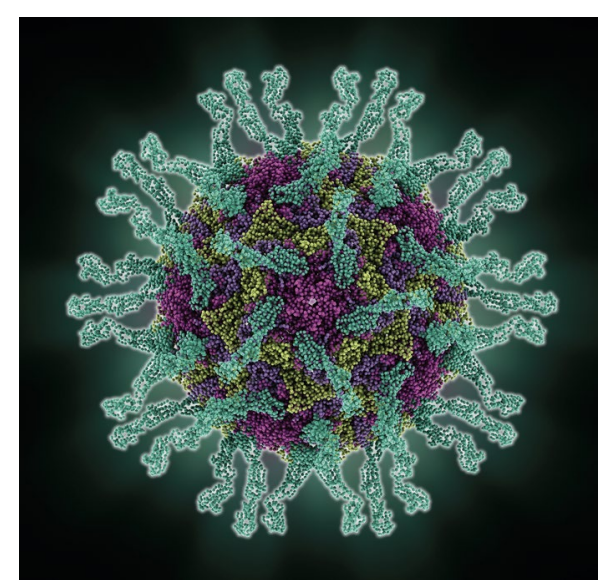

Credit: Laguna Design/Science Source

Following synchronized global withdrawal of the oral poliovirus vaccine type 2 (OPV2), outbreaks of vaccine-derived poliovirus (VDPV) type 2 occur in populations at high risk.

To ensure the full elimination of poliovirus, OPV must be withdrawn to prevent outbreaks of VDPV. This was synchronously begun worldwide in April 2016 with OPV2.

By analyzing stool samples from the populace of some countries in addition to sewage samples taken from several of these countries, researchers were able to trace the success of OPV elimination in
118 countries in total. While the rate of detection of vaccine virus declined overall, nine VDPV2 outbreaks were reported in areas associated with low coverage of immunization and low levels of population immunity. Prevention of further outbreaks in these areas is a priority.

https://doi.org/10.1038/s41591-018-0228-y

\section{GENE THERAPY}

\section{Gene editing for Duchenne muscular dystrophy}

Science https://doi.org/10.1126/science.aau1549 (2018).

The deltaE50-MD dog model of Duchenne muscular dystrophy (DMD) can be treated with systemic delivery of CRISPR gene editing components that restore the expression of the dystrophin gene.

DMD is characterized by progressive muscle degeneration and atrophy and is caused by mutations in the dystrophin gene that result in its decreased expression. It has been shown that the gene can be targeted with CRISPR-Cas9 to restore its expression in mice and muscle stem cells.

The deltaE50-MD dog model of DMD has a mutation in the dystrophin gene that corresponds with a mutational hotspot in humans. Researchers were able to restore its expression in this model using CRISPRCas9, showing the potential for translation in humans.

HS

https://doi.org/10.1038/s41591-018-0225-1
IMMUNE SYSTEM

\section{Early immune development Cell 174, 1277-1292.e14 (2018)}

Despite early differences in composition, the immune system of preterm and term children converges to a similar structure in early life.

Previous epidemiology data suggest that early-life environmental exposure shapes an individual's susceptibility to immunemediated diseases. Furthermore, a greater understanding of the composition of the neonatal immune system would help prevention and treatment of infection.

Scientists in Sweden were able to sample the cord blood and take four samples of neonatal blood from 100 children in the first 3 months of life. At birth, the immune cell and protein composition was unique to each child, but these converged onto a shared trajectory that seemed to be driven by microbial interactions.

https://doi.org/10.1038/s41591-018-0226-0

\section{SYNTHETIC BIOLOGY \\ Live bacterial therapies for metabolic disease}

Nat. Biotechnol. 36, 857-864 (2018).

The genetic disease phenylketonuria (PKU) prevents affected individuals from metabolizing phenylalanine; this metabolic function can be returned to the gut with engineered bacteria.

The inability of individuals with phenylketonuria to metabolize phenylalanine results in its accumulation in the brain and blood, neurological deficits and emotional and cognitive problems. It is currently treated with a prescription diet in conjunction with therapies.

Researchers from Synlogic, Inc. engineered a bacterial strain, activated in the anaerobic environment of the gut, that was able to metabolize phenylanaline in monkeys and mice. This approach shows promise for translation to humans and potentially also for treatment of other metabolic disorders.

https://doi.org/10.1038/s41591-018-0229-x

Hannah Stower 